Revista de Matemática: Teoría y Aplicaciones 1998 5(2) : 163-175

CIMPA - UCR - CCSS ISSN: 1409-2433

\title{
DESCOMPOSICIN DE PROCESOS DE INCREMENTOS INDEPENDIENTES EN CASI INTERVALOS
}

\author{
JAIME LOBO*
}

Recibido: 17 abril 1998

\begin{abstract}
Resumen
Se introduce la noción de proceso de incrementos independientes en casi intervalos. Son estudiadas ciertas propiedades de los puntos fijos de estos procesos así como las de la descomposición aditiva. Finalmente se establece la relación de este concepto con el clsico y se deduce una propiedad de la variación total de los procesos en tiempo continuo.
\end{abstract}

Palabras clave: procesos de incrementos independientes en casi intervalos; procesos de conteo asociados; puntos fijos; descomposición aditiva canónica; martingalas $L^{2}$-regulares en casi intervalos; condiciones de regularidad de las trayectorias; variación total; nearby processes.

\begin{abstract}
We introduce the notion of independent increment in quasi-intervals. Some properties of the fixed points of these processes are studied, as well as those of the aditive decomposition. Finally, we establish the relation between this concept with the classical one and we deduce a property of the total variation of continuous-time processes.
\end{abstract}

Keywords: independent increment processes in quasi-intervals; associated counting processes; fixed points; canonical aditive decomposition; $L^{2}$-regular martingales in quasiintervals; regularity conditions of trajectories; total variation; nearby processes.

AMS Subject Classification: 60G20, 60G44.

${ }^{*}$ CIMPA, Escuela de Matemtica, Universidad de Costa Rica, 2060 San Jos, Costa Rica 


\section{Introducción}

Los procesos de incrementos independientes históricamente han sido siempre de particular interés en la teoría de procesos estocásticos, y entre ellos se encuentran casos tan importantes como el proceso del movimiento browniano, el de Poisson y muchos otros. Diferentes enfoques se han usado para estudiar la estructura de estos procesos, comenzando por las más clásicas (teoría de leyes infinitamente divisibles) hasta aquellos que usan las técnicas del cálculo estocástico. En cualquiera de estos enfoques se presentan dificultades que muchos han tratado de simplificar por otras vías (el autor de este trabajo lo hizo para un caso particular en [6]). Desde hace algunos años se han propuesto las ideas del análisis no estándar para tratar los problemas en tiempo continuo en general. Una de las ideas más importantes de este enfoque es la de poder tratar los procesos en tiempo continuo a a partir de sus versiones en tiempo discreto recurriendo para ello a un espacio finito de probabilidades y de un proceso indexado por un casi intervalo de la recta. Siguiendo este esquema se ha llegado a obtener versiones discretas de procesos en tiempo continuo: estudio del movimiento browniano según Anderson, el cálculo estocástico, las difusiones, etc. (ver por ejemplo [3], [7], [8]). En este trabajo propongo un estudio de este estilo para los procesos de incrementos independientes, limitándome a algunos aspectos generales que no agotarían, sobra decirlo, las posibilidades de ulteriores desarrollos.

En la versión finita que trataré a continuación nos damos un espacio finito $(\Omega, P)$ de probabilidades, y un casi intervalo $T$ de la recta, es decir un conjunto finito de puntos de $\mathbb{R}$ $t_{0}=a<t_{1}<\ldots<t_{n}=b$ con la propiedad de que los puntos contiguos son infinitamente cercanos. Llamamos longitud de $T$ al valor $b-a$. Si $t+d t$ denota el punto de $T$ contiguo a $t$ a la derecha, entonces para cualquier proceso $X=\left(X_{t}, t \in T\right)$ en $(\Omega, \mathrm{P})$ indexado por $T$ llamamos el incremento en $t$ a $d X_{t}=X_{t+d t}-X_{t}$. Se supone dada una filtración en $(\Omega, P)$, es decir una sucesión creciente $F=\left(F_{t}, t \in T\right)$ de álgebras de variables aleatorias. Si $F_{t}$ denota el álgebra de variables generada por $\left(X_{u}, u \leq t\right)$ decimos que es la filtración natural de $X$. Decimos que un proceso $X$ es de $F$ incrementos independientes $(F-\mathrm{PII})$ si para cada $t$ la variable $X_{t}$ pertenece a $F_{t}$ y el incremento $d X_{t}$ es independiente de cualquier variable de $F_{t}$. Si las variables $\left(d X_{t}, t \in T, t<b\right)$ de un proceso $X$ son independientes entre ellas entonces $X$ es $F$-PII para la filtración natural $F$ de $X$.

Estudiaremos ciertas propiedades fundamentales de los $F$-PII sobre casi intervalos, la más importante relacionada con la descomposición del proceso original en la suma de otros procesos F-PII con buenas propiedades (parte previsible y martingala asociada al proceso). Se mostrará cómo esta está relacionada con las propiedades de regularidad de las trayectorias. Finalmente se explicará la relación entre $F-$ PII sobre casi intervalos y los procesos de incrementos independientes clásicos. Algunas ideas de estos resultados las he presentado en trabajos anteriores, más exactamente para los procesos puntuales en tiempo discreto con la propiedad $F$-PII en [4] y [5], de los que me serviré más adelante.

El marco formal adoptado para el análisis no estándar es el de la "Internal Set Theory" (IST), cuya exposición general puede hallarse en [1]. La teoría no estándar de espacios de probabilidad finitos está basada en [2] y [9], de la que se recordarán algunos resultados cada vez que sea necesario. Se usará la terminología y notación estándar siguientes: un número real $x$ es infinitesimal si $|x| \leq a$ para todo $a>0$ estándar. Es llamado ilimitado 
si $|x|>a$ para todo real a estándar. Si $x, y$ son reales denotamos: $x \approx y$ si $x-y$ es infinitesimal, $x \approx \infty$ si $x$ es ilimitado, $x \ll \infty$ si $x$ no es ilimitado positivo.

\section{Algunos resultados sobre la regularidad de funciones en casi intervalos}

Se considera un casi intervalo $T$. Nos interesa estudiar las propiedades de las trayectorias de los procesos estocásticos sobre $T$, para lo cual es necesario obtener algunos resultados sobre funciones reales definidas en $T$. Remito a [1], [8] ó [9] para los conceptos no estándar relacionados con estas funciones. Consideramos aquí las propiedades externas (referidas a una función $f$ definida sobre el casi intervalo $T$ ) siguientes:

$P_{1}: f$ es de fluctuación limitada en $T$ si para cada $\epsilon \gg 0$ no existe $k$ entero ilimitado tal que $f$ posea $k \varepsilon$ - fluctuaciones en $T$.

$P_{2}: f$ es continua en $t$ si para todo $s \approx t, s, t$ en $T$, se tiene $f(t) \approx f(t)$.

$P_{3}: f$ es de variación limitada en $T$ si $\sum_{s \in T}\left|d f_{t}\right|$ es limitada.

Observemos que si $T$ es de longitud limitada la propiedad $P_{1}$ se cumple para funciones continuas en todo T. Para esto establecemos primero el lema:

Lema 1 Si $T$ es de longitud limitada entonces no existe un conjunto en $T$ de cadinalidad ilimitada de puntos no infinitamente cercanos

Prueba: Supongamos que existe tal conjunto, que por ser finito podemos ordenar como $s_{0}<s_{1}<\ldots<s_{p}$, con $P$ entero ilimitado. Como para cada $i$ la cantidad $s_{i+1}-s_{i}$ es $\gg 0$,el mínimo de ellas entre todas las $i$ es un real $\alpha \gg 0$. Por tanto la cantidad $s_{p}-s_{0}$, que se expresa como $\sum_{0 \leq i<p} s_{i+1}-s_{i}$, es mayor que $p \alpha$. Pero $p \alpha$ es ilimitado y a fortiori lo es $s_{p}-s_{0}$. Pero esto contradice la hipótesis pues $s_{p}-s_{0}$ es menor que la longitud de $T$, que es limitada.

Supongamos entonces que $f$ sea continua en $T, T$ de longitud limitada. Si no fuera de fluctuación limitada existirían un $\varepsilon \gg 0$ y puntos $s_{1}<s_{2}<. .<s_{p}, p$ ilimitado, tales que $\left|f_{s_{i+1}}-f_{s_{i}}\right| \geq \varepsilon$. Por el lema 1 para algún $i: s_{i+1} \approx s_{i}$, y la relación $\left|f_{s_{i+1}}-f_{s_{i}}\right| \geq \varepsilon$ implica que $f$ no es continua en $s_{i}$ y por lo tanto no es continua en todo $T$. La trayectoria $f$ debe ser entonces de fluctuación limitada tal como se había afirmado.

Daremos algunos resultados generales sobre discontinuidades de funciones crecientes. Se dice que una función $f$ definida en $T$ posee una $\varepsilon$ - discontinuidad en $t$ si para un $s \approx t$ se tiene $\left|f_{s}-f_{t}\right| \geq \varepsilon$. El primer resultado establece que si la función crece mucho posee $\varepsilon$ -discontinuidades de todos los órdenes si el intervalo es pequeño.

Teorema 1 Sea $T$ de longitud limitada, $f$ creciente en $T$ tal que $f_{b} \approx \infty$, y todos sus incrementos son infinitesimales. Entonces para todo real positivo limitado $\varepsilon$ existe una $\varepsilon$-discontinuidad limitada de $f$ en $T$. 
Prueba: Basta probarlo para $\varepsilon$ limitado no infinitesimal. Sea $n$ el entero más grande tal que $f_{b} \geq n \varepsilon$. Entonces $n$ es limitado. Si $p \leq n$, definimos $t_{p}$ en $T$ como el menor $t$ en $T$ tal que $f_{t+d t} \geq p \varepsilon$. Entonces como $f_{t p}<p \varepsilon$ y $d f_{t p} \approx 0$ se obtiene: $f_{t p} \approx p \varepsilon$. Por lo tanto los puntos $t_{p}, p \leq n$, forman una sucesión estrictamente creciente de puntos en $T$. Pero siendo $T$ de longitud limitada del lema 1 se deduce que existen $i \approx j, i<j$ con $t_{i} \approx t_{j}$ y a fortiori $t_{i} \approx t_{i+1}$. Pero por lo anterior $f_{t_{i+1}}-f_{t_{i}} \approx \varepsilon$, por lo que $f_{t i+1}-f_{t i}$ es limitado y como $\varepsilon, \varepsilon / 2$ no son inf. cercanos se deduce que $f_{t i+1}-f_{t i}>\varepsilon / 2$.

Para los teoremas siguientes sigo de cerca el estudio sobre discontinuidades fijas de martingalas en casi intervalos desarrollada en el capítulo 11 de [2]. Asimismo echamos mano del principio externo del menor elemento: si $A$ es una propiedad (externa o interna) sobre los enteros, y si para algún $n$ entero estándar $A$ se cumple entonces existe un $m$ estándar tal que $A$ se cumple para $m$ y no se cumple para los enteros menores que $m$.

Teorema 2 Sea $f$ definida en $T$, creciente y limitada en $T$. Entonces para cada $\varepsilon \gg 0$ existe un conjunto limitado de puntos $s_{0}, s_{1}, \ldots, s_{p}$ en $T$, no infinitamente cercanos dos a dos, tal que sit es $\varepsilon$-discontinuidad de $f$ entonces para algún $i: t \approx s_{i}$.

Prueba: Sean $s_{1}, s_{1}, \ldots, s_{p}$ en $T$, no infinitamente cercanos dos a dos, que son $\varepsilon$-discontinuidades de $f$. Entonces existen casi intervalos $T_{i}, 1 \leq i \leq p$,de longitudes infinitesimales, no disjuntos dos a dos, con $s_{i} \in T_{i}$, y tales que para cada $i$ el incremento de $f$ en los extremos de $T_{i}$, denotado $d f_{T_{i}}$, es $\geq \varepsilon$. Pero entonces:

$$
p \varepsilon \leq \sum_{1 \leq i \leq p} d f_{T_{i}} \leq f(b)-f(a)
$$

y por lo tanto $p \leq(f(b)-f(a)) / \varepsilon$, donde el miembro derecho es limitado por hipótesis. Entonces existe $n$ entero limitado tal que el resultado del teorema no se cumple para este $n$. Por el principio externo del menor elemento debe existir un entero limitado $k+1$ tal que el resultado se cumple para $k$ y no para $k+1$. El conjunto de discontinuidades asociado a este $k$ responde al resultado esperado.

Se deduce el corolario siguiente, en donde se aplica el principio sucesional siguiente: si $A(n, x)$ es una fórmula interna o externa tal que existe para todo estándar $n$ un $x$ tal que $A(n, x)$, entonces existe una sucesión $n \rightarrow x_{n}$, tal que $A\left(n, x_{n}\right)$ para $n$ estándar.

Corolario 1 Bajo las hipótesis del teorema 2, existe una sucesión de puntos $t_{0}, t_{1}, \ldots, t_{n}, \ldots$ en $T$ tal que sit es discontinuidad de $f$ entonces $t \approx t_{i}$ para un estándar $i$.

Prueba: Un punto $t$ de $T$ es dicontinuidad de $f$ si y solo si es una $1 / k$ - discontinuidad de $f$ para algún $k$ entero estándar. Por el teorema 2 existe un conjunto $F_{k}$ de $T$ de cardinalidad limitada, de puntos no inf. cercanos dos a dos y tal que si $t$ es $1 / k$ discontinuidad de $f$ pero no $1 / l$ discontinuidad de $f$ para ningún $l<k$,entonces $t$ es infinitamente cercano a un punto de $F_{k}$. Por el principio sucesional existe una sucesión $k \rightarrow F_{k}$ tal que lo anterior se cumple para $k$ estándar. Sea $i$ no estándar y sea $t_{0}, t_{1}, \ldots, t_{m}$ igual a $\bigcup_{k \leq i} F_{k}$, definiendo $t_{k}$ para $k>m$ de manera arbitraria. Entonces esta sucesión satisface las conclusiones del teorema. 


\section{Procesos puntuales asociados a un F-PII; aplicaciones al estudio de las discontinuidades fijas}

Consideramos un espacio finito $(\Omega, P)$, una filtración $F$, un casi intervalo $T$, y un proceso $X$ con la propiedad $F$-PII.

Para una función $f$ de variable real definimos el proceso $S(f(d X))$ por $S(f(d X))_{a}$ $=0, d S(f(d X))_{t}=f\left(d X_{t}\right)$. Así por ejemplo si $f(x)=x, S(f(d X))$ coincide con $X-X_{a}$. Nos interesará particularmente los casos siguientes:

- las funciones de truncamiento $f(x)=x^{(\varepsilon)}=x 1_{\{|x|>\varepsilon\}}$. El proceso $S\left(d X-d X^{(\varepsilon)}\right)$ (resp. $S\left(d X^{(\varepsilon)}\right)$ ) da en cada $t$ la suma de los incrementos mayores (resp. menores) en valor absoluto que $\varepsilon$ en los tiempos anteriores a $t$

- las funciones indicadoras $f(x)=1_{\{|x|>\varepsilon\}}$, donde $\varepsilon \geq 0$. El proceso $S(f(d x))$ vale en cada $t$ el número de incrementos mayores en valor absoluto que $\varepsilon$ anteriores a $t$. Es un proceso puntual sobre $T$ (ver [5]), en el sentido de que los incrementos solo toman los valores 0 ó 1 . Convenimos en llamar $N_{\varepsilon}$ a este proceso.

- la función valor absoluto $f(x)=|x|$. El proceso $S(|d X|)$ es llamado proceso variación total de $X$.

Nótese que se cumple $d\left(S\left(d X-d X^{(\varepsilon)}\right)\right)_{t}=d X_{t} d N_{\varepsilon, t}$ en todo $t$.

Teorema 3 Para toda función $f$ el proceso $S(f(d X))$ es $F-P I I$ sobre T. Si $X$ es de fluctuación limitada c.s. se cumple que para $\varepsilon \gg 0$, los procesos $S\left(d X-d X^{(\varepsilon)}\right) y N_{\varepsilon}$ son c.s. de fluctuación limitada sobre $T$, y además $E\left(N_{\varepsilon, t}\right) \ll \infty$ si $t-a$ es limitado.

Prueba: Siendo el incremento en $t$ de $S(f(d X))$ una función de $d X_{t}$ se sigue que es independiente de cualquier variable de $F_{t}$, de donde el primer resultado. Si $X$ es de fluctuación limitada c.s. y $\varepsilon \gg 0$ necesariamente $N_{\varepsilon, t}$ es limitado c.s. en cada $t$ pues de otra manera existiría un número ilimitado de $\varepsilon$-fluctuaciones de $X$ sobre $T$.

La relación $d\left(S\left(d X-d X^{(\varepsilon)}\right)\right)_{t}=d X_{t} d N_{\varepsilon, t}$ muestra que lo mismo ocurre para $S\left(d X-d X^{(\varepsilon)}\right)$. El último resultado es consecuencia del teorema 3 en [5].

Suponemos la condición adicional de que $X$ es c.s. de fluctuación limitada. Un punto $t$ de $T$ es una discontinuidad fija de $X$ si $X$ no es c.s. continua en $t$. En virtud del teorema 7.2 en [2] y su corolario esto significa que existe $h \approx 0$ y $\varepsilon \gg 0$ tal que

$$
P\left(\operatorname{máx}_{u:|u-t| \leq h}\left|X_{t}-X_{u}\right| \varepsilon\right) \gg 0 .
$$

Nos interesa caracterizar las discontinuidades fijas de $X$. Aunque no es posible tratar las discontinuidades fijas de $X$ como un conjunto se pueden para estos obtener propiedades interesantes, análogas a las obtenidas para las martingalas (ver cap. 11 en [2]).

Denotaremos discontinuidad fija por $d . f$.

Si $X$ es c.s. continua en $t$ es necesario que c.s. para todo $s \approx t:\left|d X_{s}\right| \approx 0$. Diremos entonces que $t$ es una discontinuidad fija de salto (d.f.s.) de $X$ si no se cumple que c.s. para todo $s \approx t:\left|d X_{s}\right| \approx 0$. 
Lema 2 Si $t$ es d.f.s. de $X$, existe $\varepsilon \gg 0$ tal que $t$ es discontinuidad de la función $E N_{\varepsilon}$.

Prueba: Por hipótesis debe existir un $\varepsilon \gg 0$ tal que con probabilidad no infinitesimal $\left|d X_{s}\right|>\varepsilon$ para $s$ en un cierto intervalo infinitesimal de $t$. Esto significa precisamente que el proceso $N_{\varepsilon}$ posee una $d$.f. en $t$. Pero una $d . f$. de un proceso puntual PII es d.f. de su función esperanza (teo. 4 en [5]) y se concluye.

Teorema 4 Sea $T$ de longitud limitada. Existe una sucesión de puntos $t_{0}, t_{1}, \ldots, t_{n}, \ldots$ en $T$ tal que si $t$ es d.f. de salto de $X$ entonces $t \approx t_{i}$ para un estándar $i$.

Prueba: Por el lema 2 si $t$ es $d . f . s$. de $X$ entonces existe $m$ estándar tal que $t$ es discontinuidad de la función $E\left(N_{1 / m}\right)$, siendo $E\left(N_{1 / m}\right.$ limitada en $T$ (teorema 3$)$ existe por el corolario 1 una sucesión $t_{0}^{(m)}, t_{1}^{(m)} \ldots, t_{n}^{(m)}, \ldots$ en $T$ cumpliendo las propiedades del corolario para la función $E\left(N_{1 / m}\right)$. Entonces toda d.f.s. de $X$ es infinitamente cercana a un $t_{n}^{(m)}$ con $m, n$ estándar. Por el principio sucesional existe una sucesión $m \longrightarrow E_{m}$ tal que para $m$ estándar $E_{m}$ coincide con $t_{0}^{(m)}, t_{1}^{(m)}$...Considere una biyección estándar de $\mathbb{N}$ en $\mathbb{N} \times \mathbb{N}: k \rightarrow(i(k), j(k))$ y la sucesión $t_{0}, t_{1} \ldots, t_{h} \ldots$ definida por $t_{n}=t_{j(n)}^{i(n)}$.

Entonces esta cumple con las propiedades buscadas.

Del teorema anterior y del teorema 21.8 de [2] obtenemos un resultado que expresa que las d.f.s. de $X$ son "pocas" en el sentido de la medida:

Teorema 5 Sea $T$ de longitud limitada. Para cada $\varepsilon \gg 0$ existe un entero $n$ e intervalos $\mathbb{I}_{i}, i=1, \ldots, n$, tales que la suma de sus longitudes es menor que $\varepsilon$, y cada d.f.s. de $X$ está en uno de los $\mathbb{I}_{i}, i=1, \ldots n$, para un $i$ estándar. Además cada d.f.s. en $\mathbb{I}_{i}$, i estándar, se halla a una distancia no infinitesimal de los extremos de este intervalo.

\section{Descomposición de un F-PII}

Consideramos un espacio finito $(\Omega, P)$, una filtración $F$, un casi intervalo $T$.

\subsection{Resultados generales}

Existe una forma canónica de asociar a cada proceso definido en $T$ una martingala de la filtración considerada. Se define la parte previsible de un proceso $X$ sobre $T$ al proceso $X^{p}$ definido por $d X_{t}^{p}=E_{t}\left(d X_{t}\right)$. La martingala asociada a $Y$ es simplemente $X-X^{p}$, y la denotamos por $X^{\wedge}$. Si $X$ es una $F$ - martingala, $X^{2}$ es una submartingala de $F$. La parte previsible de $X^{2}$ es denotada $[X]$ y cumple $d[X]_{t}=E_{t}\left(d\left(X_{t}\right)^{2}\right)=E_{t}\left(\left(d X_{t}\right)+2 X_{t} d X_{t}\right)=$ $E_{t}\left(\left(d X_{t}\right)^{2}\right)$. Es inmediato entonces lo siguiente:

Teorema 6 Sea $X$ un F-PII.

a) Se tiene $d X_{t}^{p}=E\left(d X_{t}\right)$, o sea su parte previsible es determinística. En este caso la martingala asociada $X^{\wedge}$ es PII sobre $T$. 
b) Si $X$ es además F-martingala: $\left.d[X]_{t}=E d X_{t}^{2}\right)$, es decir la varianza de $d X_{t}$. Para $s<t$ se cumple $[X]_{t}-[X]_{s}=\left\|X_{t}-X_{s}\right\|_{2}^{2}$.

Llamamos función esperanza de $X$, a la función $E(X)$ en $T$ definida por $E(X){ }_{t}$ $=E\left(X_{t}\right)$ y por función varianza de $X$, denotada $\operatorname{Var}(X)$, a la función en $T$ definida por $\operatorname{Var}(X)_{t}=\operatorname{Var}\left(X_{t}\right)$. En particular para cada t se tiene: $d \operatorname{Var}(X)_{t}=\operatorname{Var}\left(d X_{t}\right)$, $d(E X)_{t}=E\left(d X_{t}\right)$. El teorema 6 implica que un $F-P I I$ es la suma de su función esperanza y de martingala PII. Con el fin de hallar propiedades más finas para la descomposición necesitaremos establecer algunos resultados sobre las martingalas que gozan de esta propiedad. El resultado que sigue da condiciones de integrabilidad para martingalas de incrementos no muy grandes. Recordemos que una variable $Z$ es $L^{p}$ integrable según Loeb-Nelson si $|Z|^{p}$ es $L^{1}$ (ver cap. 8 en [2]). Es necesario primeramente un lema sencillo cuya prueba omito:

Lema 3 Si $Y$ es variable centrada, entonces para todo e $>0$ se tiene: $\operatorname{Var}(Y) \geq \operatorname{Var}(Y-$ $\left.Y^{(e)}\right), \operatorname{Var}(Y) \geq \operatorname{Var}\left(Y^{(e)}\right)$.

Teorema 7 Sea $X$ una $F$ - martingala PII nula en en a tal que $\operatorname{Var}(X)_{b} \ll \infty$, y tal que existe $c$ real positivo limitado con $\left|d X_{t}\right| \leq c$ para todo $t$. Entonces:

a) Para todo $t$ la variable $X_{t}$ es $L^{2}$ integrable según Loeb-Nelson y c.s. X es de fructuación limitada.

b) Para todo $\varepsilon>0$ las martingalas asociadas a $S\left(d X-d X^{(\varepsilon)}\right)$ y $S\left(d X^{(\varepsilon)}\right)$ son $L^{2}$, c.s.de fluctuación limitada.

Prueba:

a) Toda martingala $L^{2}$ es c.s. de fluctuación limitada (teorema 22.3 en [2]). Basta entonces mostrar que $X$ es $L^{2}$ integrable según Loeb- Nelson. Para esto usamos el hecho de que si para un $p \gg 1$ se tiene $\mathrm{E}(|Z| p) \ll \infty$ entonces $Z$ es $L^{1}$ (ver [2]). El resultado del teorema se obtendría si para un $p \gg 2$ se tiene $E\left(\left|X_{b}\right|^{p}\right) \ll \infty$, y cierto para todo $t$ pues $E\left(\left|X_{t}\right|^{p}\right.$ es creciente en $t$. Mostremos que esto es cierto para $p=4$. Toda martingala siendo de incrementos ortogonales, $X$ siendo además suma de los incrementos independientes centrados $d X_{t}$, al expander $X_{b}^{4}$ obtenemos:

$$
E\left(X_{b}^{4}\right)=3 \sum_{s \neq t} E\left(d X_{s}^{2} d Y_{t}^{2}\right)+\sum_{s} E\left(d X_{s}^{4}\right)
$$

El segundo término lo mayoramos por $c^{2} \sum_{s} E\left(d X_{s}^{2}\right)=c^{2} \operatorname{Var}(X)_{b} \ll \infty$. El primer término es también limitado pues por la hipótesis PII:

$$
\begin{aligned}
\sum_{s \neq t} E\left(d X_{s}^{2}\right) E\left(d X_{t}^{2}\right) & =\sum_{s} E\left(d X_{s}^{2}\right) \sum_{t: t \neq s} E\left(d X_{t}^{2}\right) \\
& =\sum_{s} E\left(d X_{s}^{2}\right)\left(\operatorname{Var}(X)_{b}-E\left(d X_{s}^{2}\right)\right) \\
& =\operatorname{Var}(X)_{b}^{2}-\sum_{s}\left(E\left(d X_{s}^{2}\right)\right)^{2}
\end{aligned}
$$


y basta acotar $\sum_{s}\left(E\left(d X_{s}^{2}\right)\right)^{2}$ por $c^{2} \operatorname{Var}(X)_{b}$

b) Si se cumplen las condiciones de a) para estas martingalas tendríamos b). De los teoremas 3 y 6 las martingalas asociadas a $S\left(d X-d X^{(\varepsilon)}\right)$ ), $S\left(d X^{(\varepsilon)}\right)$ (que según lo anterior se escriben $\left.\left.S\left(d X-d X^{(\varepsilon)}\right)\right)^{\wedge}, S\left(d X^{(\varepsilon)}\right)^{\wedge}\right)$ son $P I I$. Por el lema 3 es claro que $\operatorname{Var}(X)$ es mayor que $\operatorname{Var}\left(S\left(d X-d X^{(\varepsilon)}\right)^{\wedge}\right), \operatorname{Var}\left(S\left(d X^{(\varepsilon)}\right)^{\wedge}\right)$ en todos los puntos de $T$, por lo que los valores de estas funciones varianza son limitados en $T$. Finalmente los incrementos de estas martingalas están mayoradas en valor absoluto por $2 c$ en cada punto.

\subsection{Descomposición del proceso sin discontinuidades fijas}

En lo sucesivo se considera un proceso $X F$-PII sin discontinuidades fijas (como en 3-1 la notación d.f. significa discontinuidad fija). Supondremos además que el casi intervalo $T$ es de longitud limitada. Decimos que una martingala $X F-\mathrm{PII}$ es $L^{2}$-regular en $T$ si para todo $t X_{t}$ es $L^{2}$, c.s. $X$ es de fluctuación limitada y la función varianza de $X$ es continua en $T$. Decimos que $X$ es incrementos uniformemente limitados (IUL) si existe un real positivo limitado $c$ tal que para todo $(\omega, t)$ se cumple $\left|d X_{t}\right| \leq c$ para todo $t$ en $T$.

Deducimos primero un resultado importante para martingalas PII de incrementos no muy grandes.

Teorema 8 Sea $X$ martingala con la propiedad IUL. Se cumple:

a) Xes $L^{2}$-regular en $T$

b) Para todo $\varepsilon>0$ las martingalas asociadas a $S\left(d X-d X^{(\varepsilon)}\right)$ y $S\left(d X^{(\varepsilon)}\right)$ son $L^{2}$ regulares $y$ no poseen d.f.

c) Para $\varepsilon \approx 0$, la martingala asociada a $S\left(d X^{(\varepsilon)}\right)$ es continua en todo $T$.

\section{PRUEBA:}

a) Por definición la función $\operatorname{Var} X_{t}$ es $E\left(X_{t}\right)^{2}$, que es creciente. Si mostramos que $\operatorname{Var}(X)_{b} \ll \infty$, todas las condiciones del teorema 7 se cumplen y de este se deduce que para todo $t X_{t}$ es $L^{2}$ y que c.s. $X$ es de fluctuación limitada. En cuanto a la continuidad de $\operatorname{Var}(X)$ notemos que si $s, t$ puntos de $T$ son infinitamente cercanos, no habiendo d.f. se tiene $X_{t}-X_{s} \approx 0$ c.s.y como de lo anterior $X_{t}-X_{s}$ es $L^{2}$, del teorema de Lebesgue se obtiene $\operatorname{Var}(X)_{t}-\operatorname{Var}\left(X_{s}\right)=\left\|X_{t}-X_{s}\right\|_{2}^{2} \approx 0$. Entonces $\mathrm{X}$ es $L^{2}$ regular en $T$.

Notemos primero que para cada $t$ la variable $\left(d X_{t}\right)^{2}$ es c.s. infinitamente cercana a 0 (pues no hay d.f.) y es $L^{1}$ integrable por la condición $\left|d X_{t}\right| \leq c$. Por el teorema de Lebesgue (versión no estándar) se tiene $E\left(\left(d X_{t}\right)\right)^{2} \approx 0$. Pero $E\left(\left(d X_{t}\right)^{2}\right)=$ $d(\operatorname{Var}(X))_{t}$ por lo que todos los incrementos de la función varianza de $X$ son infinitesimales.

Supongamos por contradicción que $\operatorname{Var}(X)_{b} \approx \infty$. En este caso se cumplen las condiciones del teorema 1 para la función creciente $\operatorname{Var}(X)$ por lo que existen 
$s, t$ en $T, s<t, s \approx t$, tales que $0 \ll \operatorname{Var}(X)_{t}-\operatorname{Var}(X)_{s} \ll \infty$. El proceso $X-X_{s}$ es una martingala PII sobre el casi intervalo $T \cap[s, t]$ de función varianza $\operatorname{Var}(X)-\operatorname{Var}(X)_{s} \ll \infty$. Como $\operatorname{Var}(X)_{t}-\operatorname{Var}(X)_{s} \ll \infty$ el proceso $X-X_{s}$ satisface en $T \cap[s, t]$ las condiciones del teorema 7 y por lo tanto $X-X_{s}$ es $L^{2}$. Pero además $X_{t}-X_{s}$ es c.s. infinitamente cercana a 0 (no hay d.f.) y por el teorema de Lebesgue $\left\|X_{t}-X_{s}\right\|_{2}^{2} \approx 0$,relación que contradice el hecho de que $\operatorname{Var}(X)_{t}-\operatorname{Var}(X)_{s}$ no es infinitesimal.

b) Siendo $X L^{2}$-regular (resultado a)), satisface las condiciones del teorema 7 . De b) de este teorema deducimos que las martingalas asociadas a $S\left(d X-d X^{(\varepsilon)}\right.$ y $S\left(d X^{(\varepsilon)}\right)$ son $L^{2}$, c.s. de fluctuación limitada. Probemos que sus funciones varianzas son continuas en $T$ lo que basta hacerlo para una de ellas, digamos $S\left(d X^{(\varepsilon)}\right)^{\wedge}$. Llamemos $Z=$ $S\left(d X^{(\varepsilon)}\right)^{\wedge}$. Del lema 3 aplicado a las variables $d X_{t}$ se tiene que el incremento de $\operatorname{Var} X$ en dos puntos cualesquiera de $T$ es mayor que el incremento respectivo de $\operatorname{Var}(Z)$ en esos puntos, y como $\operatorname{Var}(X)$ es continua en $T$ se deduce esta misma propiedad para $\operatorname{Var}(Z)$.

La martingala $Z=S\left(d X^{(\varepsilon)}\right)^{\wedge}$ es entonces una martingala $L^{2}$ continua en la métrica $L^{2}$. Pero una martingala $L^{1}$ continua en la métrica $L^{2}$ no posee d.f. (teorema 11.4 en [2] ,lo que resulta cierto entonces para $Z$. La prueba para $S\left(d X-d X^{(\varepsilon)}\right)^{\wedge}$ es análoga.

c) Por b) la martingala $Z=S\left(d X^{(\varepsilon)}\right)^{\wedge}$ es $L^{2}$ y por esto se cumple la propiedad de que c.s. para todo $\varepsilon \gg 0$ no existen dos $\varepsilon$ - fluctuaciones en cualquier intervalo de $T$ en donde el crecimiento de $\operatorname{Var}(Z)$ sea infinitesimal (teorema 13.1 en [2]). La $L^{2}$-regularidad en $T$ implica además que en cualquier intervalo de longitud inifinitesimal el crecimiento de $\operatorname{Var}(Z)$ es infinitesimal. Resulta entonces que para c.s. para todo $\varepsilon \gg 0$ no existen dos $\varepsilon$ - fluctuaciones en cualquier intervalo de $T$ de longitud infinitesimal. De aquí se deduce la continuidad c.s.de $Z$. En efecto: los incrementos de $Z$ son inifinitesimales en todo $(\omega, t)$ pues $\left|d Z_{t}\right|=\left|d X_{t}^{(\varepsilon)}-E\left(d X_{t}^{(\varepsilon)}\right)\right| \leq$ $\left|d X_{t}^{(\varepsilon)}\right|+\left|E\left(d X_{t}^{(\varepsilon)}\right)\right| \leq 2 \varepsilon$. Si para $s, t, s \approx t$, se tuviera $\left|Z_{t}-Z_{s}\right| \gg 0$,debería existir un $u$ entre $s, t$, tal que $\left|Z_{t}-Z_{u}\right| \gg 0,\left|Z_{u}-Z_{s}\right| \gg 0$, sea dos fluctuaciones no infinitesimales en un intervalo de longitud infinitesimal, que contradice lo anterior.

Un resultado análogo al anterior sin la condición martingala es:

Teorema 9 Sea $X$ c.s. de valores limitados en todo $T$ y con la propiedad IUL. Entonces su función esperanza es continua y limitada en $T$ y su martingala asociada es $L^{2}$ - regular en $T$.

Prueba: Pasando al espacio producto $(\Omega \times \Omega, P \times P)$ se considera un proceso $X^{\prime}$ independiente de $X$ y de misma ley (definiendo por ejemplo $X^{\prime}\left(\omega, \omega^{\prime}\right)=X\left(\omega^{\prime}\right)$ ) y se define luego $Z=X-X^{\prime}$. El proceso $X$ goza de las mismas propiedades que las de $X^{\prime}$. Entonces $Z$ también las cumple. Pero $X, X^{\prime}$ teniendo ambos la misma ley se cumple $E\left(d Z_{t}\right)=0$, es decir $Z$ es además una martingala. Entonces del teorema 8 se deduce que $\operatorname{Var}(Z)_{b} \ll \infty$, o sea $\operatorname{Var}(Z)_{t} \ll \infty$ para todo $t$. Pero $\operatorname{Var}\left(Z_{t}\right)=\operatorname{Var}\left(X_{t}\right)+\operatorname{Var}\left(X_{t}\right)$, por lo tanto $\operatorname{Var}\left(X_{t}\right) \ll \infty$. En particular las variables $X_{t}$ son todas $L^{1}$ y no habiendo d.f. la función 
esperanza de $X$ es continua. Por lo tanto la martingala asociada a $X$ satisface entonces las condiciones del teo.8 del cual se concluye.

Corolario 2 Sea $X$ de fluctuación casi siempre limitada. Entonces para todo real positivo $\varepsilon, 0 \ll \varepsilon \ll \infty$, la función esperanza de $S\left(d X^{(\varepsilon)}\right)$ es continua y limitada y la martingala asociada a $S\left(d X^{(\varepsilon)}\right)$ es $L^{2}$-regular en $T$.

Prueba: Sea $Z=S\left(d X^{(\varepsilon)}\right)$. Basta verificar que las condiciones del teorema 9 se cumplen para $Z$. Primeramente sabemos por el teorema 3 que $Z$ es un PII de fluctuación limitada. Entonces c.s. los valores de $Z$ en todo $T$ son limitados. En efecto, dado un real $c, 0 \ll c \ll$ $\infty$, definimos recurrentemente los tiempos $t_{p}$ en $T$ como: $t_{0}$ el más pequeño $s$ en $T$ tal que $\left|Z_{s+d s}-Z_{a}\right| \geq c$,si $p \geq 1, t_{p}$ es el más pequeño $s \geq t_{p-1}$ tal que $\left|Z_{s+d s}-Z_{t_{p-1}}+d t_{p-1}\right| \geq c$ $\left(t_{p}=b\right.$ si tal $s$ no existe).

El número total de tiempos así definidos debe ser c.s. limitado. Sea $t$ en $T$ y tomemos $p$ tal que $t_{p} \leq t<t_{p+1}$. Como para todo $p$ se tiene $\left|d Z_{t_{p}}\right| \leq \varepsilon \mathrm{y}\left|Z_{t_{p}}-Z_{t_{p-1}+d t_{p-1}}\right|<c$ entonces:

$$
\left|Z_{t}\right| \leq \sum_{q \leq p}\left|Z_{t_{q}}-Z_{t_{q-1}+d t_{q-1}}\right|+\sum_{q<p}\left|d Z_{t_{q}}\right|+\left|Z_{t}-Z_{t_{p}}\right|
$$

donde el miembro derecho es limitado pues cada suma posee un número limitado de términos mayorados por una constante limitado, y el último es menor que $c$ por definición.

Verifiquemos que no tiene d.f. Por definición $\left|d Z_{t}\right|=\left|d X_{t}^{(\varepsilon)}\right| \leq \varepsilon$ para todo $t$ y $\omega$. Una d.f. de $X-Z$ es una del proceso puntual $N_{\varepsilon}$ que es d.f.s. de $X$. Por lo tanto $X-Z$ no posee d.f. y lo mismo sucede para $Z$ como suma de procesos con esta propiedad. Así entonces de donde la conclusión.

Consideremos el proceso variación total de $X$ definido en la sección 2. Denotémoslo por $V T X$. Deducimos de lo anterior algunas propiedades de este proceso:

Teorema 10 Sea $X$ de fluctuación casi siempre limitada. Entonces para todo real positivo $\varepsilon, 0 \ll \varepsilon \ll \infty$, el proceso variación total asociado a $Z=S\left(d X^{(\varepsilon)}\right)$ es en t: c.s.limitado si $E(V T Z)_{t} \approx \infty$; c.s. ilimitado si $E(V T Z)_{t} \approx \infty$. Su parte martingala es $L^{2}$ en $t$.

Prueba: Llamemos $Z=S\left(d X^{(\varepsilon)}\right)$. Primeramente notemos que para toda variable $Y$ : $\operatorname{Var}(Y) \geq \operatorname{Var}(|Y|)$. Si $V T Z \wedge, Z^{\wedge}$ son las martingalas asociadas a $V T Z, Z$ respectivamente, sabemos por definición de éstas que $d\left(\operatorname{Var}\left(V T Z^{\wedge}\right)\right)_{t}=\operatorname{Var}\left(d(\operatorname{VTZ})_{t}\right)$, $d\left(\operatorname{Var}\left(Z^{\wedge}\right)\right)_{t}=\operatorname{Var}\left(d Z_{t}\right)$. Como además $d(V T Z)_{t}=\left|d Z_{t}\right|$ de lo anterior se obtiene $d\left(\operatorname{Var}\left(V T Z^{\wedge}\right)\right)_{t} \leq d\left(\operatorname{Var}\left(Z^{\wedge}\right)\right)_{t}$. Por lo tanto $\operatorname{Var}\left(V T Z^{\wedge}\right)_{t} \leq \operatorname{Var}\left(Z^{\wedge}\right)_{t}$ y dado que $\operatorname{Var}\left(Z^{\wedge}\right)_{t} \ll \infty$ (corolario 2) se tiene $\operatorname{Var}\left(V T Z^{\wedge}\right)_{t} \ll \infty$. Como los incrementos de $V T Z^{\wedge}$ son en valor absoluto menores que $2 \varepsilon$, se cumplen las condiciones del teorema 7 , por lo que $V T Z_{t}^{\hat{t}}$ es $L^{2}$. En particular $V T Z_{t}=V T Z_{t}-E\left(V T Z_{t}\right)$ es c.s. limitada, de donde los primeros asertos. 


\section{Relación entre PII clásicos y en casi intervalos}

En esta sección me propongo demostrar que la noción de PII en casi intervalo es un verdadero análogo discreto de la noción clásica. Por otro lado daré un ejemplo de cómo deducir un resultado para los procesos clásicos a partir de los obtenidos en las secciones anteriores.

En la versión del ANS que seguimos es posible tratar procesos clásicos estándar y versiones discretas sin recurrir a las llamadas extensiones (ultraproductos, ultralímites) que se usaron en los inicios del ANS. La versión discreta buscada es llamada en [2] un nearby process que en el caso del tiempo continuo se formaliza así: dado un proceso estándar, $Y_{t}, t$ $\in I, I$ intervalo de $\mathbb{R}$, definido en un espacio estándar $(\Omega, A, P)$, decimos que $Y_{t}^{\prime}, t \in T, T$ casi intervalo que contiene a los puntos estándar de $I$, definido en el espacio finito $\left(\Omega^{\prime}, P^{\prime}\right)$, subespacio de $(\Omega, A, P)$, es nearby si la relación $\sum_{t \in T}\left|Y_{t}-Y_{t}^{\prime}\right| \approx 0$, salvo en un evento de probabilidad infinitesimal. Se puede precisar diciendo que para cada $\varepsilon \approx 0$ positivo el proceso $X$ se puede escoger de tal manera que

$$
(*): \sum_{t \in T}\left|Y_{t}-Y_{t}^{\prime}\right| \leq \varepsilon
$$

se cumple fuera del evento de probabilidad infinitesimal. Se prueba que para un proceso $Y$ siempre existe un nearby $Y^{\prime}$. En la construcción de $Y^{\prime}$ no se garantiza que la propiedad PII sea conservada, sin embargo:

Teorema 11 Si $Y$ es un PII, entonces existe $Y^{\prime}$ nearby con la misma propiedad.

Prueba: Considere $T$ casi intervalo de $I$ conteniendo todos los estándar de $I$. Se construye de la misma manera que en un nearby del proceso de los incrementos de $Y$ sobre $T:\left((d Y)_{t}^{\prime}, t \in T\right)$ es nearby a $\left(d Y_{t}, t \in T\right)$. La relaciòn $(*)$ se cumple para un cierto $\varepsilon$. Por construcción misma de este proceso se tiene que $(d Y)_{t}^{\prime}=f\left(d Y_{t}\right)$ para todo $t$, donde $f$ es una función de variable real. Entonces por definición misma de PII las variables $\left((d Y)_{t}^{\prime}, t \in T\right)$ son independientes. Escoja $\varepsilon$ de tal manera que $\varepsilon|T|$ sea infinitesimal y defina $Y_{t}^{\prime}=\sum_{s \in T, s<t}(d Y)_{s}^{\prime}$. Como fuera de un evento raro

$$
\left|Y_{t}-Y_{t}^{\prime}\right| \leq \sum_{s \in T, s<t}\left|(d Y)_{s}^{\prime}-d Y_{s}\right| \leq \varepsilon
$$

entonces

$$
\sum_{s \in T, s<t}\left|Y_{s}^{\prime}-Y_{s}\right| \leq \varepsilon|T| \approx 0
$$

fuera de ese evento raro. El proceso $Y_{t}^{\prime}$ es pues un nearby de $Y$ y es además PII.

Por los llamados teoremas de equivalencia (teorema A.7 y A.3 en [2]) muchas propiedades del proceso inicial $Y$ se conservan para su nearby $Y^{\prime}$. La propiedad conservada para $Y^{\prime}$ es en general una propiedad externa sobre las trayectorias de $Y^{\prime}$. Consideramos por ejemplo las propiedades $P_{1}, P_{2}, P_{3}$ citadas en la sección 1.Entonces se cumplen las equivalencias: 
- c.s. $Y$ es lag-lad (sin discontinuidades de segunda especie) en I $\Leftrightarrow$ c.s. la trayectoria de $Y^{\prime}$ es $P_{1}$.

- c.s. Y es continuo en $t$ ( $t$ estándar) $\Leftrightarrow$ c.s.la trayectoria de $Y^{\prime}$ es $P_{2}$ en $t$.

- c.s. $Y$ es continuo en $I \Leftrightarrow$ c.s.la trayectoria de $Y^{\prime}$ es $P_{2}$ en todo punto de $T$.

- c.s. $Y$ es de variación acotada en $I \Leftrightarrow$ c.s. la trayectoria de $Y^{\prime}$ es $P_{3}$ en $T$.

En cada uno de estos resultados el enunciado de la izquierda se interpreta de manera clásica, mientras que en el derecho se trata de una propiedad externa sobre las trayectorias de $Y^{\prime}$ que se cumple según la definición no estándar de propiedad externa casi siempre cierta. Recordemos que una propiedad externa $P$ es cierta casi siempre si para cada $\varepsilon>0$ estándar existe un evento $N$ con $P\left(N^{c}\right) \leq \varepsilon$ y $P$ se cumple en $N$. Además para cada una de las propiedades externas $P_{i}$ existen formas equivalentes para expresar que se cumplen casi siempre.

Estudiemos ahora la variación total de un proceso PII en tiempo continuo bajo el supuesto de que c.s. sus trayectorias son continuas:

Teorema 12 Sea $Y$ un F-PII en tiempo continuo sobre un intervalo I de $\mathbb{R}$, tal que c.s. la trayectoria de $Y$ es continua en I. Entonces solo existen dos alternativas: c.s. $Y$ es de variación acotadas en $I$ ó c.s. $Y$ es de variación infinita en $I$.

Prueba: Por transfer basta suponer que $Y$ es estándar. Consideremos su nearby process $Y^{\prime}$ en un casi intervalo $T$ de $I$ que goza según el teorema 11 de la propiedad PII. Por el teorema de equivalencia anterior $Y^{\prime}$ es a su vez c.s. continuo. Sea un real positivo $\varepsilon, 0 \ll \varepsilon \ll \infty$, y definamos $Z=S\left(d Y^{\prime(\varepsilon)}\right)$. Entonces $Z$ es un PII sobre $T$, y siendo $Y^{\prime}$ c.s. continuo en $T$ el proceso $Z$ coincide con $Y^{\prime}$ en todo $T$ casi siempre. Gracias al teorema 10 sabemos que $Z$ ó es c.s. de variación limitada, o es c.s. de variacin ilimitada y por lo tanto lo mismo se cumple para $Y^{\prime}$.

En el caso de que $Y^{\prime}$ sea c.s. de variación limitada sabemos del teorema de equivalencia que $Y$ es c.s. de variación finita.

Basta probar ahora que si $Y^{\prime}$ es c.s.de variación limitada entonces $Y$ es c.s. de variación infinita. Este resultado no se prueba en [2], por lo que debe establecerse. Para esto definimos para cada conjunto finito $F$ de $I$ y cada entero $k$ el conjunto

$$
\phi_{k, F}=\left\{\sum_{t \in F^{\prime}}\left|Y_{t+d t}-Y_{t}\right| \leq k\right\}
$$

siendo $F^{\prime}$ el conjunto sin su último elemento, y $t+d t$ denotando el sucesor de $t$ en $F$. En un espacio de probabilidades apropiado (como el descrito en [2]) los $\phi_{k, F}$ son además eventos por lo que es posible asignarles probabilidades. Notemos que el evento $\Gamma$ definido por:

$$
\Gamma=\bigcup_{K} \bigcap_{F} \phi_{k, F}
$$


es precisamente el evento donde $Y$ es de variación finita. Puesto que $\phi_{K, F}$ es creciente en $k$ y decreciente en $F$, en virtud del teorema A.6 en [2] se tiene:

$$
P(\Gamma)=\sup _{K} \inf _{F} P\left(\phi_{k, F}\right)
$$

Supongamos entonces que $Y$ no es c.s. de variación finita. Entonces $P(\Gamma)>0$ y siendo la probabilidad de un evento estándar esta probabilidad es además no infinitesimal. Por transfer existe un estándar $\varepsilon>0$ y en entero estándar $k$ tal que $\inf _{F} P\left(\phi_{k, T}\right) \geq \varepsilon$. En particular $P\left(\phi_{k, F}\right) \geq \varepsilon$. Sea $N$ el evento de probabilidad infinitesimal tal que la relación $\left.{ }^{*}\right)$ se cumple en $N^{c}$. Entonces $\phi_{k, T} \cap N^{c}$ es de probabilidad no infinitesimal y sobre este evento la variación total de $Y^{\prime}$ es menor o igual que $k$, o sea de variación ilimitada. Entonces $Y^{\prime}$ no es de variación c.s. limitada. Usando la contrapositiva obtenemos el resultado.

\section{Comentarios finales}

En este trabajo se ha realizado un estudio bastante sistemático de los procesos que he llamado de incrementos independientes sobre casi intervalos. De particular atención ha sido el problema de la descomposición aditiva estudiada en la sección 3. El resultado acerca de las propiedades de la variación de un PII en tiempo continuo (teorema 12), muestra el interés del estudio de los procesos en casi intervalos como análogos discretos de los procesos en tiempo continuo.

No obstante quedan planteados muchos otros problemas que no he abordado en este artículo. Es necesario por ejemplo un estudio más completo de las discontinuidades fijas de la sección 2, pues en realidad solo se examinó sistemáticamente las discontinuidades fijas de salto. Por otro lado es pertinente preguntarse sobre otras consecuencias de los teoremas de descomposición para PII en casi intervalos para los de tiempo continuo. ¿Se puede por ejemplo reestablecer de alguna manera las propiedades de las leyes de procesos estocásticamente continuos, por ejemplo la ley de Lévy-Khinchin? El autor ha presentado respuestas a estas preguntas en el caso particular de los procesos puntuales PII, estableciendo el carácter poissoniano de las leyes de los incrementos ([4]). Conjeturo que las ideas desarrolladas en [4] podrían ser generalizadas para tratar el caso general que he planteado.

\section{Referencias}

[1] Nelson, E. (1977) "Internal Set Theory: a new approach to nonstandar analysis ", Bulletin of the American Mathematical Society 83(6).

[2] Nelson, E. (1987) Radically Elementary Probability Theory. Princeton University Pres.

[3] Stroyan, B. (1986) Foundations of Infinitesimal Stochastic Analysis. North Holland, Amsterdam.

[4] Lobo, J. (1995) "Estudio en tiempo discreto de los procesos puntuales de incrementos condicionalmente independientes", Revista de Matemática: Teoría y Aplicaciones 2(2): 9-16. 
[5] Lobo, J. (1995) "Una noción de proceso puntual en tiempo discreto", Revista de Matemática: Teoría y Aplicaciones 2(1): 17-25.

[6] Lobo, J. (1985) Processus Accroissements Indépendants et Méthode des Semimartingales. Tesis de Doctorado de 3er. ciclo, Universidad de Paris 6.

[7] Lindstrom (1986) "Nonstandar constructions of diffusions and related processes", Memorias del 1er. Congreso Mundial de la Sociedad Bernoulli, Vol. 1, Scientific Press.

[8] Albeverio (1986) Nonstandar Methods in Stochastic Analysis and Mathematical Physics. Academic Press, New York.

[9] Chuaquí, R. (1991) Truth, Possibility and Probability. North Holland, Amsterdam. 